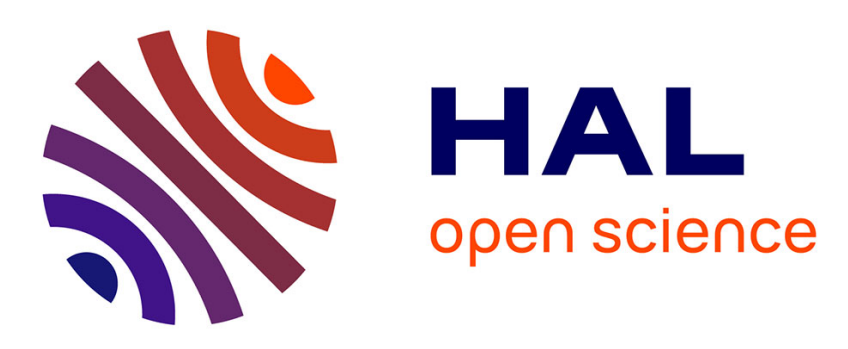

\title{
Adsorption of organic compounds at the surface of Enceladus' ice grains. A grand canonical Monte Carlo simulation study
}

\author{
Julien Joliat, Antoine Patt, Jean Marc Simon, Sylvain Picaud
}

\section{To cite this version:}

Julien Joliat, Antoine Patt, Jean Marc Simon, Sylvain Picaud. Adsorption of organic compounds at the surface of Enceladus' ice grains. A grand canonical Monte Carlo simulation study. Molecular Simulation, 2022, pp.19-30. 10.1080/08927022.2021.1900571 . hal-03366327v2

\section{HAL Id: hal-03366327 \\ https://hal.science/hal-03366327v2}

Submitted on 10 Oct 2022

HAL is a multi-disciplinary open access archive for the deposit and dissemination of scientific research documents, whether they are published or not. The documents may come from teaching and research institutions in France or abroad, or from public or private research centers.
L'archive ouverte pluridisciplinaire HAL, est destinée au dépôt et à la diffusion de documents scientifiques de niveau recherche, publiés ou non, émanant des établissements d'enseignement et de recherche français ou étrangers, des laboratoires publics ou privés. 


\title{
Adsorption of organic compounds at the surface of Enceladus' ice grains. A grand canonical Monte Carlo simulation study
}

\author{
Julien Joliat ${ }^{a}$, Antoine Patt ${ }^{a}$, Jean-Marc Simon ${ }^{b}$ and Sylvain Picaud ${ }^{a}$ \\ ${ }^{a}$ Institut UTINAM, UMR 6213, CNRS, Université Bourgogne Franche-Comté, \\ Besançon, France \\ ${ }^{b}$ Laboratoire Interdisciplinaire Carnot de Bourgogne, UMR 6303, CNRS, \\ Université de Bourgogne Franche-Comté, Dijon, France \\ sylvain.picaud@univ-fcomte.fr
}

\begin{abstract}
In this paper, we characterise the adsorption of ethylene, propanol and hexanal molecules on crystalline ice by grand canonical Monte Carlo simulations performed at $236 \mathrm{~K}$, a temperature which is typical of some Enceladus' environments. We show that at low coverage of the ice surface, the adsorption of propanol and hexanal is driven by the interaction of these molecules with the ice phase and, as a consequence, the adsorbed molecules lie more or less parallel to the ice surface. On the other hand, upon saturation, the adsorbate-adsorbate interactions become more and more important and the molecules tend to become tilted with respect to the surface, the aliphatic chain pointing towards the gas phase, while the polar head of propanol and hexanal molecules always stays attached to the ice surface, irrespective of the coverage. By contrast, the ethylene molecules do not show any strong affinity for the ice surface because of the corresponding weak binding energy. These results are in good agreement with the major features provided by the available experiments on similar systems.
\end{abstract}

KEYWORDS: Enceladus, ice, adsorption, trace gases, simulation

\section{Introduction}

Enceladus is an active moon of Saturn which hides a global ocean of liquid salty water beneath its icy crust [1]. This moon has been widely studied by the Cassini-Huygens mission a few years ago, that evidenced, among other discoveries, jets of icy particles from the internal ocean, mixed with a variety of simple organic compounds $[2,3]$. The plumes thus formed, extend hundreds of miles into space. Although approximately $5-10 \%$ of the emitted icy grains escapes and enters the Saturn's E ring, most of the matter they contain finally falls back onto the surface [4].

Since the ocean in Enceladus supplies the jets, knowing the exact plume composition would allow a better quantification of the abundance of organics at Enceladus, which is a prerequisite to understand its ocean's chemistry and, thus, its possible habitability [5]. However, the organic molecules present in the vapour phase of the plumes are in contact with the icy grains that are concomitantly ejected. They also interact, before ejection, with the ice walls of the cracks through which the jets have erupted. As a consequence, these organics/ice interactions have an important impact on the measured composition of the plumes [6], due to the differences in depletion vs release mechanisms that are driven by the characteristics of the involved species [7]. Indeed, adsorption of organic molecules on ice surfaces has been widely studied in the Earth's context, where it has been recognised as an important phenomenon able to modify the fate of various species in the troposphere as well as on the ice mantles at the surface $[8,9]$.

In a recent study, Bouquet et al. have developed an analytical adsorption/desorption model to quantify the influence of the ice grains and ice walls on the plume composition [5].

This model requires the knowledge of the binding energy of the adsorbed compounds to water ice, most of the corresponding values being based on available experimental values, obtained on Earth's conditions [5]. The general conclusion of this study is that, in conditions relevant to Enceladus, adsorption on ice strongly segregates between compounds that are trapped or released by the ice surface, as a function of their binding energy. According to Bouquet et al., compounds with binding energy greater than $-0.5 \mathrm{eV}$ are thus negligibly present at the ice surface, whereas those with binding energy less than $-0.7 \mathrm{eV}$ dominates at the surface. Compounds with intermediate binding energies may also stay at the surface in some situations, if the competitive adsorption with those of lower energy allows [5]. As a consequence, the preponderance of a compound at the ice surface is not directly correlated to its abundance in gas phase, and this has to be taken into account in the conclusions that can be drawn from the corresponding measurements.

A thorough characterisation of the uptake processes 
of organics on ice is thus of fundamental interest to adequately assess the fate of these species on Enceladus. However, although the adsorption of trace gases on ice surfaces has been widely characterised experimentally in the past decades in various contexts, including interstellar ices $[7,8,9,10,11,12,13,14]$, some compounds of interest for Enceladus have not been studied yet, or their interaction with ice has been characterised in conditions that are not transferable to Enceladus. This implies that their binding energy to ice is not accurately known [6, 15]. Moreover, fundamental questions regarding the location of the adsorbed molecules, their orientation, the number of hydrogen bonds they form at the ice surface as well as their ability to form single or multi-layers on ice often remain unanswered.

All these points can be theoretically tackled by means of atomistic simulations, given that the model chosen to represent the organic/water interactions, accurately reproduces known experimental properties $[16,17,18]$. As far as the structure and the energy of the adsorption process are concerned, the Grand Canonical Monte Carlo (GCMC) method has been shown to be particularly suitable to provide detailed direct insights of the studied system, at the molecular level [18]. Indeed, GCMC simulations allow the theoretical determination of the adsorption isotherm, i.e. the surface coverage as a function of the partial pressure at a given temperature. In addition, the results of the simulations can be statistically analysed for fixed values of the partial pressure, and give access to the average binding energy and the distribution of the molecular orientations at the surface.

Thus, for more than 15 years, we have used the GCMC method to model, at the molecular scale, the interaction between ice and various organic molecules (see Ref. [18] and reference therein). More recently, we also used the same approach to simulate the trapping of various atomic and molecular species at the surface or inside clathrate hydrates, another form of solid water, in the astrophysical context [19, 20, 21, 22, 23]. In these simulations, large numbers of molecules have been considered and the temperature effects have been included. This is an advantage over other methods like, e.g. optimisation methods or quantum calculations that are usually restricted to a limited number of molecules, the adsorption of which being most often characterised at $0 \mathrm{~K}$.

Here, we have performed GCMC simulations to investigate the behaviour at the surface of crystalline $\mathrm{I}_{\mathrm{h}}$ ice of three molecules, namely ethylene $\left(\mathrm{C}_{2} \mathrm{H}_{4}\right)$, propanol $\left(\mathrm{C}_{3} \mathrm{H}_{8} \mathrm{O}\right)$ and hexanal $\left(\mathrm{C}_{6} \mathrm{H}_{12} \mathrm{O}\right)$ at $236 \mathrm{~K}$, i.e. a temperature relevant for the ice grains and the ice walls that are in contact with organic compounds in the cracks near the surface of Enceladus [5]. These molecules have been selected among others because, to the best of our knowledge, their adsorption on ice has never been simulated before, and available experimental data indicate that their binding energy to ice $\left(\mathrm{E}_{\mathrm{b}}\right)$ ranges in the three different domains mentioned by Bouquet et al. [5], namely $\mathrm{E}_{\mathrm{b}}\left(\mathrm{C}_{2} \mathrm{H}_{4}\right)=-0.16 \mathrm{eV}$ [24], $\mathrm{E}_{\mathrm{b}}\left(\mathrm{C}_{3} \mathrm{H}_{8} \mathrm{O}\right)=-0.71 \mathrm{eV}[25]$ and $\mathrm{E}_{\mathrm{b}}\left(\mathrm{C}_{6} \mathrm{H}_{12} \mathrm{O}\right)$ $=-0.67 \mathrm{eV}[26]$. Notice that ethylene and propanol have been actually detected by the Cassini mission [15], contrarily to hexanal, the mass of which being out of the instrumental range of measurements. Nevertheless, this molecule has been taken into account in the model developed by Bouquet et al. [5] and thus deserves consideration in the present GCMC simulations.

This paper is organised as follows. In Section 2 details of the GCMC simulations are given, and the corresponding results for the three organic compounds considered here are detailed in Section 3 and discussed in Section 4. Finally, in Section 5 the main conclusions of this study are summarised.

\section{Details of the computer simula- tions at the molecular scale}

The adsorption of $\mathrm{C}_{2} \mathrm{H}_{4}, \mathrm{C}_{3} \mathrm{H}_{8} \mathrm{O}$ and $\mathrm{C}_{6} \mathrm{H}_{12} \mathrm{O}$ has been studied at the surface of crystalline hexagonal ice, at $236 \mathrm{~K}$. The algorithm of Buch et al. [27] has been used to prepare the $\mathrm{I}_{\mathrm{h}}$ phase of ice by considering water molecules arranged in 18 layers (containing 160 molecules each), along the interface normal axis (z) in such a way that adsorption on the (0001) surface of crystalline ice can be investigated. The ice solid has a proton disordered hexagonal structure that fulfils the Bernal-Fowler rules [28], with no net charge nor total dipole moment. In the GCMC simulations, this ice slab has been placed in the middle of a rectangular simulation box, the edges of which being equal to 35.926 and $38.891 \AA$ along the $\mathrm{x}$ and $\mathrm{y}$ axes, respectively. Periodic boundary conditions have been applied to the simulation box in these $(\mathrm{x}, \mathrm{y})$ directions, whereas a large vacuum above the surface along the $\mathrm{z}$ direction has been created, with three-dimensional periodicity (see Figure 1). The simulated system is thus made of a stack of non-interacting ice slabs of infinite surface area, in such a way that adsorption can be considered at the two gas/ice interfaces present in the simulation box to ensure better statistics especially when the number of adsorbed molecules is very weak. In the present simulations, the edge of the rectangular basic simulation box has been $100 \AA$ along the $\mathrm{z}$ direction, a value that represents a good compromise between a sufficiently large space separating two (non-interacting) gas/ice interfaces and a volume to be filled not too large, when the condensation pressure has been reached in the simulations. Notice that, some tests have been performed with a value of 150 
$\AA$, which did not show any noticeable differences in the corresponding results.

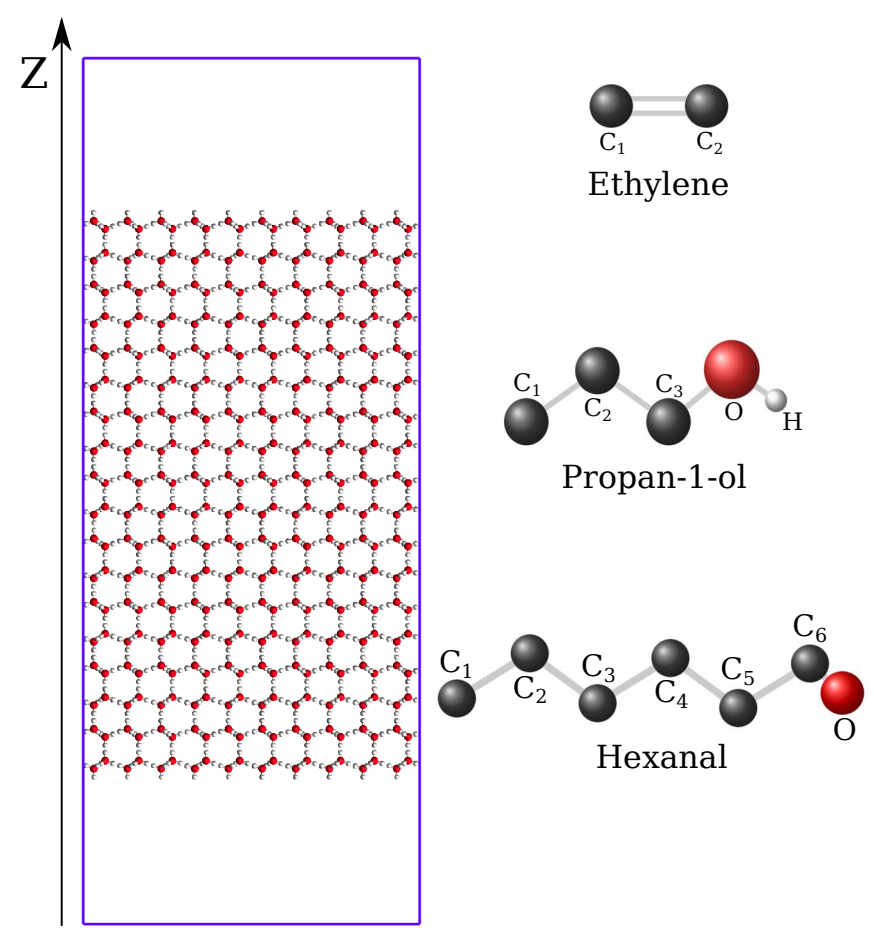

Figure 1: Visualisation of the crystalline $I_{h}$ structure of ice used in the present work. The geometry of the ethylene, propanol and hexanal molecules considered as adsorbates in the GCMC simulations is also shown on the right hand side of the figure, with the corresponding atom numbering. Oxygen, carbon and hydrogen atoms are represented as red, black, and white balls, respectively.

The water molecules have been described by the four-site TIP4P/ice model [29] which reproduces well the phase diagram of water. With this model, the melting temperature of $\mathrm{I}_{\mathrm{h}}$ ice is around $272 \mathrm{~K}$ [29], i.e. well above the temperature considered here, thus avoiding any artificial ice melting in the present GCMC simulations. Simple intermolecular potentials, based on site-site electrostatic and Lennard-Jones dispersionrepulsion contributions, have been used to represent the organic compounds. The parameters of the corresponding models have been taken from the literature and they are gathered in Table 1. Thus, ethylene has been described by an anisotropic united-atoms force field specifically parametrised to predict the equilibrium properties of various olefins [30]. Similarly, the anisotropic united-atoms AUA4 potential has been chosen to model both the propanol [31] and hexanal [32] molecules. Indeed, these interaction models have been shown to accurately represent experimental data for the thermodynamics properties of these organic compounds. Notice that, while the small ethylene molecule has been kept rigid, flexible propanol and hexanal molecules have been considered in our simulations. The usual Lorentz-Berthelot combining rules have been used to calculate cross interactions between water and the organic molecules [33]. In our GCMC simulations, the short-range Lennard-Jones interactions have been truncated using a simple cut-off equal to half the shortest box-length whereas the Ewald summation method has been used to calculate the electrostatic interactions, in accordance with the original parameterisation of the corresponding models. The details of the interaction potential models can be found in the original publications [30, 31, 32]. Monte Carlo simulations in the grand canonical ensemble have been performed using the GIBBS code [34]. The average number of particles $\langle\mathrm{N}\rangle$ in the simulation box has been calculated at constant temperature $(\mathrm{T}=236$ $\mathrm{K})$, volume $(\mathrm{V})$ and fugacity $(f)$ [21]. Different fugacity values have been used to simulate adsorption processes from vanishingly small surface coverage to the condensation situation. For each fugacity value, $10^{9}$ iteration steps have been performed to ensure the equilibration, followed by a production stage of $2.10^{8}$ steps. Data have been collected every $4.10^{4}$ steps for statistical analysis (meaning that 5000 configurations have been included in the analysis, each time). In these MC simulations, particle displacement and insertion/deletion moves have been performed with equal probabilities. For the rigid ethylene molecule, these probabilities have been $25 \%, 25 \%$ and $50 \%$, for translation, rotation, and insertion-deletion moves, respectively. For the flexible propanol and hexanal molecules, changes of the internal molecular geometry have also been considered, and the probabilities for moves have thus been set to $20 \%$ for translation, $20 \%$ for rotation, $20 \%$ for internal changes, and the remaining $40 \%$ for insertion-deletion. Notice that uniform probability of insertion has been used for the gas and solid regions, such as no bias has been introduced in the choice of the insertion sites for the organic molecules. In addition, because adsorption process was investigated, only the organic molecules have been inserted or deleted in the simulation box and, accordingly, only translations and rotations have been allowed for the water molecules.

\section{Simulation results}

\subsection{Adsorption of ethylene on crystalline ice}

Let us first detail the main properties of ethylene adsorbed on proton disordered crystalline $\mathrm{I}_{\mathrm{h}}$ ice. The adsorption isotherm, $\langle\mathrm{N}\rangle(f)$, for this species is shown in Figure 2, as obtained from the GCMC simulations at $236 \mathrm{~K}$. The obtained curve departs from zero at a fugacity value around $10^{5} \mathrm{~Pa}$ and then increases with increasing steepness in a quite narrow range of fugacity, up to the point where the condensation of ethylene occurs, at a fugacity value of about $10^{6} \mathrm{~Pa}$. Note that above the condensation pressure, the increase of the 
isotherm is simply related to the high compressibility of the ethylene fluid at high pressure, corresponding to a density increase from $455 \mathrm{~kg} / \mathrm{m}^{3}$ to $577 \mathrm{~kg} / \mathrm{m}^{3}$, between 1.6 and $101 \mathrm{MPa}$, at $236 \mathrm{~K} \mathrm{[35].}$

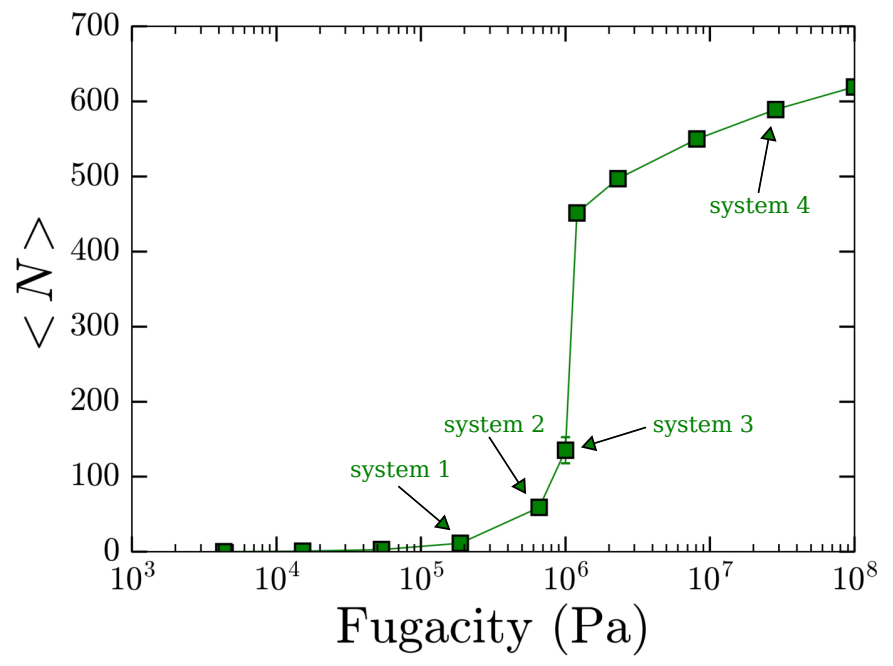

Figure 2: Average number of ethylene molecules in the basic simulation box as a function of the fugacity, as calculated from GCMC simulations performed on the ethylene $/ I_{h}$ ice system at $236 \mathrm{~K}$. The arrows indicate the systems used in the detailed analysis. Error bars are also indicated. The line connecting the points issued from the simulations only serves as a guide to the eye.

This behaviour is similar to what has been simulated for the adsorption of formaldehyde on ice [36] and might indicate that no saturated monolayer of ethylene molecules is particularly stable at the ice surface. To confirm this assumption, we have analysed the results of the GCMC simulations at three fugacity values below the condensation, corresponding to very low (System 1), medium (System 2) and high (System 3) coverages of the ice surface, and just above the condensation (System 4) (as marked by the arrows in Figure 2). As an example of analysis aiming at characterising the translational arrangement of the ethylene molecules, their number density profile $\rho(\mathrm{z})$ is shown in Figure 3(a). This number density profile gives the average number of ethylene molecules (represented by the position of their centre of mass) that are located at a given position along the ice surface normal axis $\mathrm{z}$. Notice that the density profile for the interfacial water molecules is also indicated in this figure, for reference.

In the case of System 1, all the ethylene molecules are adsorbed at the surface of ice, as the density profile nearly drops to zero beyond the $\mathrm{z}$ value of about $87 \AA$, i.e. at the distance that is about one molecular layer away from the ice surface. In systems 2 and 3, most of the ethylene molecules are again in contact with the ice phase, although a weak shoulder at larger $\mathrm{z}$ positions indicates that a second peak of the density profile starts to appear at about $\mathrm{z}=88.5$ $\AA$. Finally, in System 4, the ethylene molecules occupy (a)

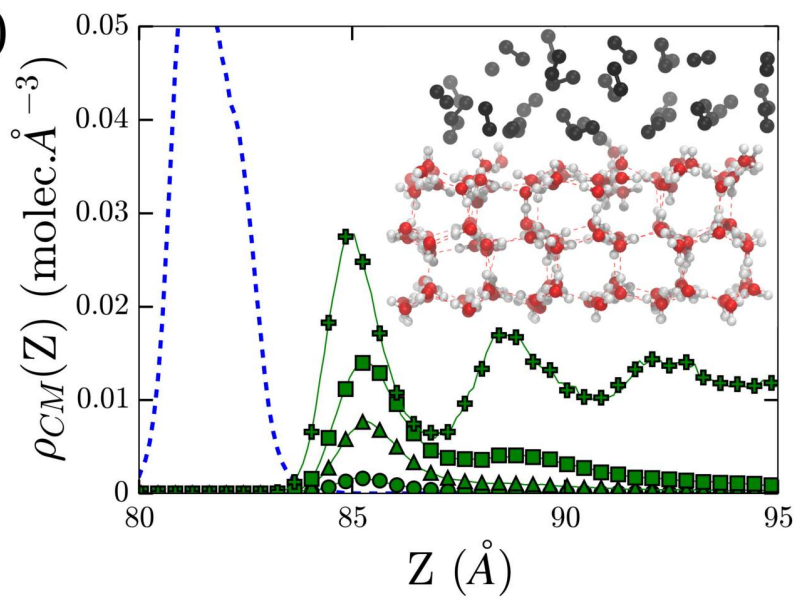

(b)

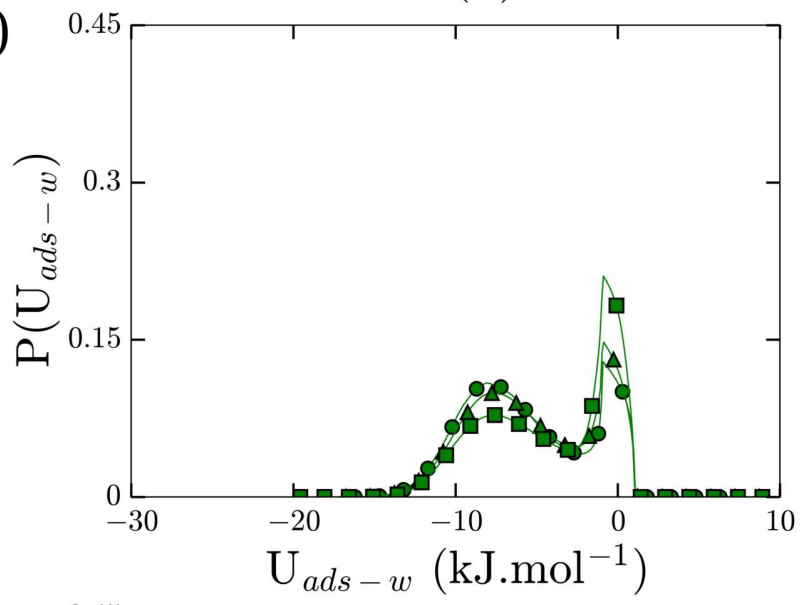

(c)

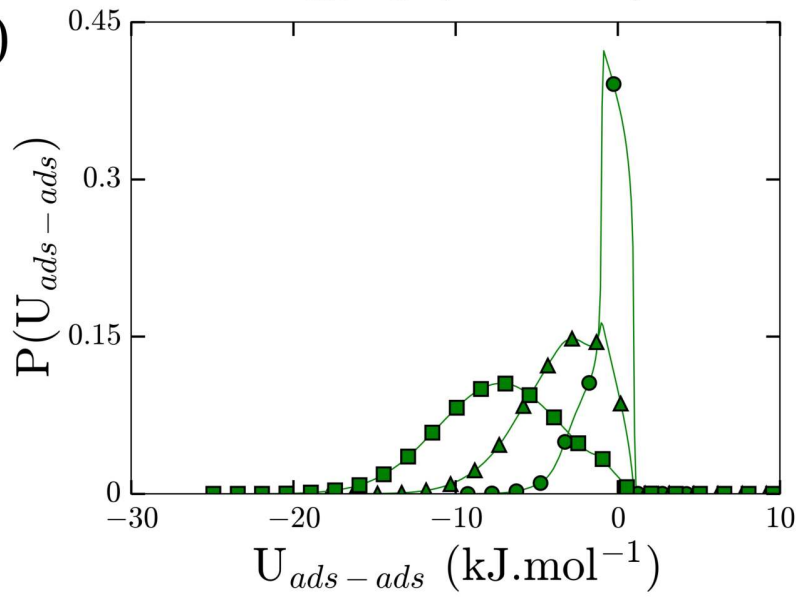

Figure 3: (a) Number density profile of the ethylene centres of mass in Systems 1 (circles), 2 (triangles), 3 (squares) and 4 (crosses), as obtained from the simulations. For reference, the outer tail of the number density profile of the water centres of mass in System 1 is also shown (dashed blue line). All profiles shown are symmetrised over the two surfaces present in the basic simulation box. (b) Distribution of the interaction energy of an adsorbed ethylene molecule with the ice phase and (c) with the other adsorbed molecules (circle, triangle and square symbols for Systems 1, 2 and 3 , respectively). A snapshot issued from the simulations of System 4 is also given in panel (a) to illustrate the geometry of the ethylene molecules at the surface of ice.

the whole empty space above the ice phase, because 3D condensation of the adsorbate has already occurred at the corresponding fugacity value. Interestingly, the intensity of the peak located at around $85 \AA$ in 
Systems 3 and 4, which corresponds to the ethylene molecules that are directly in contact with the ice surface, is much higher than the peaks located at the same position for Systems 1 and 2. This, together with the long tail of the peak observed for System 3, clearly indicates that additional layers of ethylene molecules started to build up before the first adsorption layer is completed. Note that, in this first adsorption layer, the ethylene molecules lie parallel to the ice surface, as shown by the statistical analysis of the molecular orientations (not displayed).

To characterise the energetic background of the adsorption, we have thus analysed the distributions of the interaction energy of an adsorbed ethylene molecule with all the water molecules of the ice phase, $\mathrm{U}_{\text {ads-w }}$, and that of a given ethylene molecule with all the other ethylene molecules present in the simulation box, $\mathrm{U}_{\text {ads-ads }}$ (Figure $3(\mathrm{~b}, \mathrm{c})$ ), as obtained in Systems 1,2 , and 3 .

At low surface coverage (System 1$)$, the $\mathrm{P}\left(\mathrm{U}_{\text {ads-w }}\right)$ and $\mathrm{P}\left(\mathrm{U}_{\text {ads-ads }}\right)$ distributions have their peak at around -9 and $0 \mathrm{~kJ} / \mathrm{mol}$, respectively, indicating that the interaction of ethylene with ice is very weak and that the lateral interactions within the adsorbate are vanishingly small. Consequently, for System 1, the mean value of the $\mathrm{P}\left(\mathrm{U}_{\text {ads-w }}\right)$ distribution can be regarded as an estimate of the heat of adsorption at very low surface coverages. At higher coverage (System 2), weak lateral interactions are evidenced by the shift of the peak of $\mathrm{P}\left(\mathrm{U}_{\text {ads-ads }}\right)$ to smaller (i.e. larger in magnitude) energies, which now appears at around $-4 \mathrm{~kJ} / \mathrm{mol}$. This tendency is reinforced for System 3 , where the lateral interactions increase and shift the peak of $\mathrm{P}\left(\mathrm{U}_{\text {ads-ads }}\right)$ down to about $-9 \mathrm{~kJ} / \mathrm{mol}$. However, this increase of the interaction between coadsorbed ethylene molecules does not significantly change their interaction with the ice phase, the peak of the $\mathrm{P}\left(\mathrm{U}_{\text {ads-ads }}\right)$ distribution being still observed at around $-9 \mathrm{~kJ} / \mathrm{mol}$. Note that the narrow peak at around $0 \mathrm{~kJ} / \mathrm{mol}$ in the $\mathrm{P}\left(\mathrm{U}_{\text {ads-w }}\right)$ distributions for Systems 1, 2 and 3 also reveals that some ethylene molecules are located far enough from the ice surface to not significantly interact with the water molecules, in accordance with the long tails of the peaks evidenced in the density profiles of the ethylene centres of mass (Figure 3(a)).

To summarise, the results of the statistical analysis presented above confirm that, as for formaldehyde [36], the shape of the isotherm adsorption of ethylene on ice does not evidence any formation of a stable monolayer, a feature that can be related to the weakness of the interaction between the ethylene molecules and the ice phase.

\subsection{Adsorption isotherms of propanol and hexanal on crystalline ice}

The adsorption isotherms $\langle\mathrm{N}\rangle(f)$ of propanol and hexanal, as obtained from the simulations, are plotted in Figure 4(a). In the case of propanol (red curve), this isotherm typically shows three different regions. At low fugacity values, the isotherm exhibits an increase up to about 1.0 Pa that corresponds to the building up process of the adsorption layer during which the possible adsorption sites at the ice surface are gradually occupied. Above this $f$ value, a pseudo-plateau is observed in the isotherm which indicates that the adsorption layer is close to be saturated. Indeed, large changes in the fugacity result in only a small increase (of no more than about 10\%) of the number of adsorbed molecules in this region. Finally, at $f$ value of about $35 \mathrm{~Pa}$, the isotherm exhibits a sudden jump, corresponding to the 3D condensation of propanol and above this fugacity, the box is thus entirely filled with propanol molecules. In this respect, the adsorption isotherm for propanol is rather similar to what was previously obtained for methanol [37].

The adsorption isotherm $\langle\mathrm{N}\rangle(f)$ calculated for hexanal (black curve) also exhibits three typical regions, as for propanol, although its behaviour is not exactly similar. Indeed, the adsorption isotherm for hexanal is firstly characterised by a sharp exponential increase at low fugacity value, indicating that the adsorption of an individual hexanal molecule is independent from the presence or absence of other molecules at the surface of ice. The isotherm then exhibits an increasing linear shape rather than a plateau, for fugacity values ranging typically between 0.05 and $9 \mathrm{~Pa}$. This behaviour rather seems correspond to a continuous, progressive saturation of all the adsorption sites at the ice surface, than to the stabilisation of a monolayer in a large range of fugacity values. Finally, above $9.5 \mathrm{~Pa}$, condensation of hexanal occurs, as indicated by the sudden jump observed in the isotherm at this fugacity value.

Notice that we have also checked the influence of the box size on these results by recalculating some points of the isotherms, considering now a box of edge size equal to $150 \AA$ along the $\mathrm{z}$ axis, instead of 100 A. No noticeable difference has been evidenced in the results obtained with these two different box sizes. More specially, the 3D condensation has been shown to occur at the same fugacity values in both cases and for the two species considered, thus precluding any artefact due to the size of the empty space between the ice slabs.

To further investigate the differences between propanol and hexanal behaviours at the surface of ice, their adsorption isotherms have been converted to the $\Gamma\left(f_{\text {rel }}\right)$ form, where $\Gamma$ is the surface density of the 
(a)

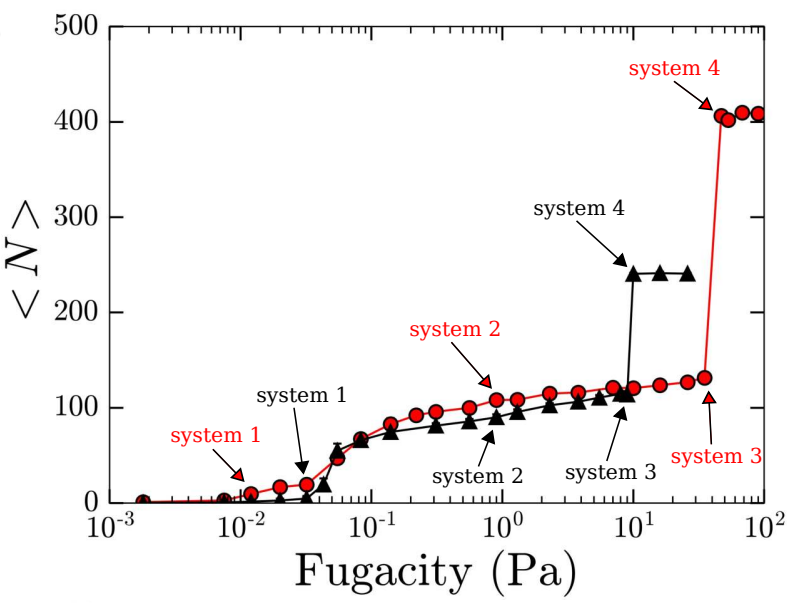

(b)

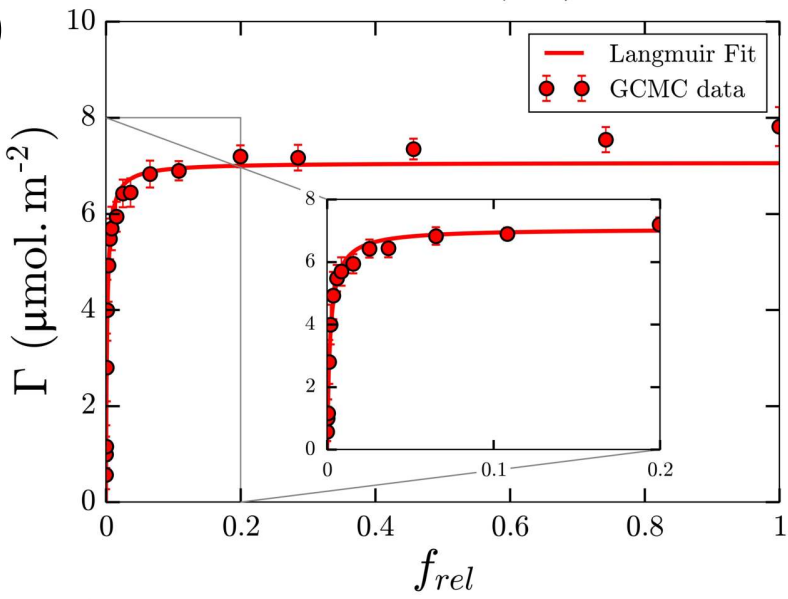

(c)

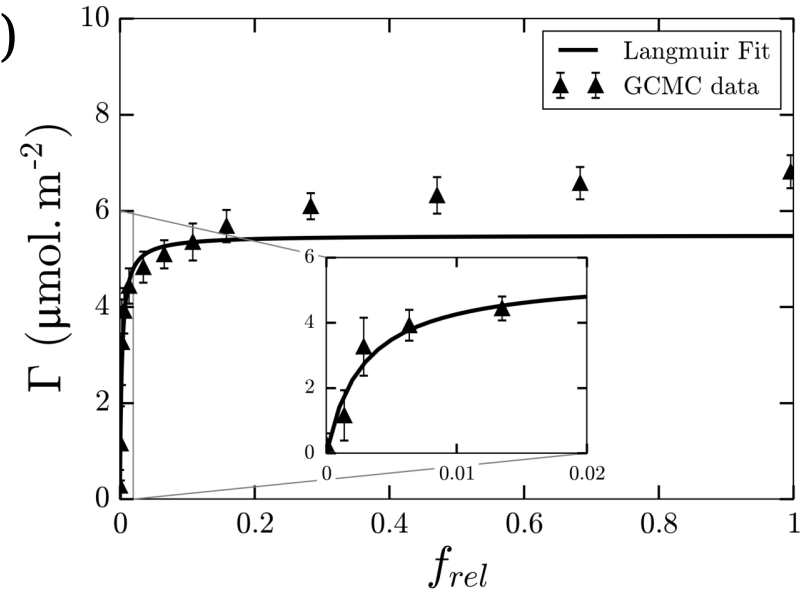

Figure 4: (a) Average number of propanol (red circles) and hexanal (black triangles) molecules in the basic simulation box as a function of the fugacity (errors bars are also indicated), as calculated from the present GCMC simulations. The arrows indicate the systems used in the detailed analyses. Lines are guide for eyes, only. Panels (b) and (c) show the corresponding curves fitted by the Langmuir equation to these data (best fit) for propanol (red) and hexanal (black), respectively. In these panels (b) and (c), inserts show the range of relative pressure in which the best Langmuir fit can be achieved from the simulation data.

adsorbed molecules expressed in $\mu \mathrm{mol} . \mathrm{m}^{-2}$, and $f_{\text {rel }}$ is the fugacity of the vapour phase $f$ normalised by the fugacity $f_{0}$ at which the $3 \mathrm{D}$ condensation is observed for the species considered. The value of $\Gamma$ has simply been calculated, up to the point where condensation occurs, as

$$
\Gamma=\frac{\langle\mathrm{N}\rangle}{2 \mathrm{XY}}
$$

where $\langle\mathrm{N}\rangle$ is the number of propanol or hexanal molecules that are adsorbed at the ice surface and $\mathrm{X}$ and $\mathrm{Y}$ are the lengths of the box edges along the $\mathrm{x}$ and $\mathrm{y}$ directions, respectively. Note that the factor 2 at the denominator accounts for the fact that, due to the periodicity of the system, the ice phase has two surfaces in the basic box. The corresponding calculated isotherms are shown in the $\Gamma\left(f_{\text {rel }}\right)$ form in Figure 4(b,c) for propanol and hexanal, respectively. Following our previous studies on alcohol [37] and aldehyde $[36,38,39]$ molecules adsorbed on ice, these two isotherms have been tentatively described in terms of the Langmuir isotherm, i.e. by the function:

$$
\Gamma=\Gamma_{\max } \frac{f_{\mathrm{rel}} \mathrm{K}}{f_{\mathrm{rel}} \mathrm{K}+1}
$$

where the parameters $\Gamma_{\max }$ and $\mathrm{K}$ are the saturated surface density and Langmuir partitioning coefficient, respectively.

As is seen from Figure 4(b), the calculated $\Gamma\left(f_{\text {rel }}\right)$ points for propanol can be well fitted by the Langmuir isotherm up to about $f_{\text {rel }}=0.1$, i.e. a fugacity range for which the lateral interactions between the adsorbed molecules seem to not play any significant role. However, at higher relative fugacity values, the simulated isotherm clearly departs from the Langmuir behaviour, as previously observed for methanol [37]. For hexanal, no satisfactory Langmuir fit can be achieved on the simulated data, except perhaps when considering the lowest relative fugacity values, only (Figure 4(c)). This could originate from larger lateral interactions within the adsorbate, even at low coverage of the ice surface. The behaviour of hexanal on ice is thus closer to that of benzaldehyde [38] than of acetaldehyde [39] and could be related to molecular size effects (here, the length of the aliphatic carbon chain).

\subsection{Analysis of the adsorption layers of propanol and hexanal on crystalline ice}

To investigate in more details the adsorption characteristics of propanol and hexanal on ice, four different values of the fugacity have been selected for which statistical analyses of the adsorbed phase have been performed. Thus, System 1, corresponding to very low fugacity value, is located at the exponentially rising part of the isotherms, where even the first molecular layer of the adsorbate is far from being saturated. The fugacity value selected for System 2 is located approximately at the position where the quasi-plateau of the propanol isotherm starts or in the middle of 
the fugacity value range where the linear part of the isotherm of hexanal is obtained. The fugacity value for System 3 is chosen to be just before the condensation occurs. Finally, System 4 corresponds to the condensed phase.

\subsubsection{Density profiles}

The density profiles of the propanol and hexanal molecules along the interface normal axis $\mathrm{z}$, as obtained in systems 1-4, are presented in Figures 5 and 6 , respectively. In these density profiles, separated analyses have been done for the positions of the molecular centres of mass and of various atoms of the molecules. All the profiles shown are averaged over the two interfaces present in the basic box. As is seen, all these profiles present typically one single peak before the condensation occurs (i.e. for Systems 1-3), the intensity of this peak increasing with the coverage of the ice surface. This indicates that the saturated adsorption layer is still monomolecular up to the point where the presence of a rather disordered condensed phase is evidenced at the highest fugacity values considered here (Systems 4). Additional information can be obtained by carefully looking at the peak positions. Indeed, for propanol the density peak of the $\mathrm{O}$ atoms is always located closer to the ice surface than that of both the centre of mass and the terminal $\mathrm{C}$ atoms, indicating that the adsorbed molecules prefer to point, on average, towards the ice surface by their $\mathrm{OH}$ group. Interestingly, the density profile for $\mathrm{H}$ atoms exhibits only one peak in the case of System 1, while two peaks are obtained for the other Systems, suggesting that both donor and acceptor hydrogen bonds with the ice surface are thus formed when the propanol coverage increases (Figure 4(d)). Moreover, increasing the propanol coverage from System 1 to System 3 leads to a small shift of both the centre of mass and $\mathrm{O}$ atom density peaks to higher distances from the ice surface (Figure 5(a,c)), while the peak position of the terminal $\mathrm{C}$ atoms density does not shift (Figure 5(b)). We can thus infer some configurational changes of the propanol molecular orientation upon coverage increase, the molecular axis straightening out on the surface when the number of propanol molecules increases at the surface.

For hexanal, the density peaks of the terminal C $\left(\mathrm{C}_{6}\right)$ and $\mathrm{O}$ atoms (Figure 6(b,c)) are always located closer to the ice surface than that of the centres of mass (Figure 6(a)), indicating that the adsorbed molecules prefer to point, on average, towards the ice surface by their aldehyde functional group. Moreover, increasing the coverage of the ice surface does not lead to any significant shift to higher distances of the density peaks of the $\mathrm{O}$ (Figure 6(c)) and terminal $\mathrm{C}$ atoms (Figure 6(b)), contrarily to the $2 \AA$ shift observed for the peaks of the density of the centres of mass (Figure 6(a)) This suggests that a reorientation of the adsorbed molecules occurs upon saturation of the adsorption layer, the molecular axis becoming less tilted with respect to the surface normal when increasing the number of adsorbed molecules, but without changing the configuration of the carbonyl group with respect to the ice surface. In other word, only the long aliphatic chain seems to be affected by the coverage increase. This is clearly confirmed by the density profile of the methyl carbon atom (labelled $\mathrm{C}_{1}$, see Figure 1) given in Figure 6(d), which exhibits a main peak at around $85.5 \AA$ in System 1, that shifts to higher values around $90 \AA$, when the coverage is increased (Systems 2-4).

These points are further discussed in a following sub-section devoted to the analysis of the molecular orientations of the adsorbed molecules at the ice surface.

\subsubsection{Energetic background of the adsorption}

In order to get insight into the energetic background of the adsorption, we have calculated the distribution of the $\mathrm{U}_{\text {ads-ads }}$ and $\mathrm{U}_{\text {ads-w }}$ contributions to the total binding energy, coming from the interaction between the adsorbed (propanol or hexanal) molecules and the water molecules of the ice phase and from the lateral interaction with the other adsorbed molecules, respectively. The corresponding $\mathrm{P}\left(\mathrm{U}_{\text {ads-w }}\right)$ and $\mathrm{P}\left(\mathrm{U}_{\text {ads-ads }}\right)$ distributions obtained in Systems 1-3 (i.e. before condensation has occurred) are shown on the top and bottom panels of Figure 7, respectively.

As is seen, at low surface coverage (i.e. in System 1 for both propanol and hexanal adsorbates) the $\mathrm{P}\left(\mathrm{U}_{\text {ads-w }}\right)$ distribution exhibits a single, rather large, peak around $-67 \mathrm{~kJ} / \mathrm{mol}$ and $-58 \mathrm{~kJ} / \mathrm{mol}$, for propanol and hexanal adsorbates, respectively, the corresponding mean values of the distribution being about $-70 \mathrm{~kJ} / \mathrm{mol}$ and $-59 \mathrm{~kJ} / \mathrm{mol}$. Notice that, as stated above for ethylene, these values can serve as an estimate for the heat of adsorption at infinitely low coverage [39].

Upon increasing the coverage of the ice surface, the peak of the $\mathrm{P}\left(\mathrm{U}_{\text {ads-w }}\right)$ distribution gradually shifts to higher energies (i.e. smaller absolute values), its maximum value being at about -41 and $-39 \mathrm{~kJ} / \mathrm{mol}$ in System 3, for propanol and hexanal molecules, respectively, as a consequence of the increasing competition of the lateral interactions between adsorbed molecules (see below). In addition, for this system that corresponds to a fugacity value just below $f_{0}$ (i.e. the value at which condensation occurs), a very small number of molecules starts to be adsorbed farther from the ice surface in the GCMC runs, as indicated by the small peak of the $\mathrm{P}\left(\mathrm{U}_{\text {ads-w }}\right)$ distributions, in the $[-10,0] \mathrm{kJ} / \mathrm{mol}$ range. 
(a)

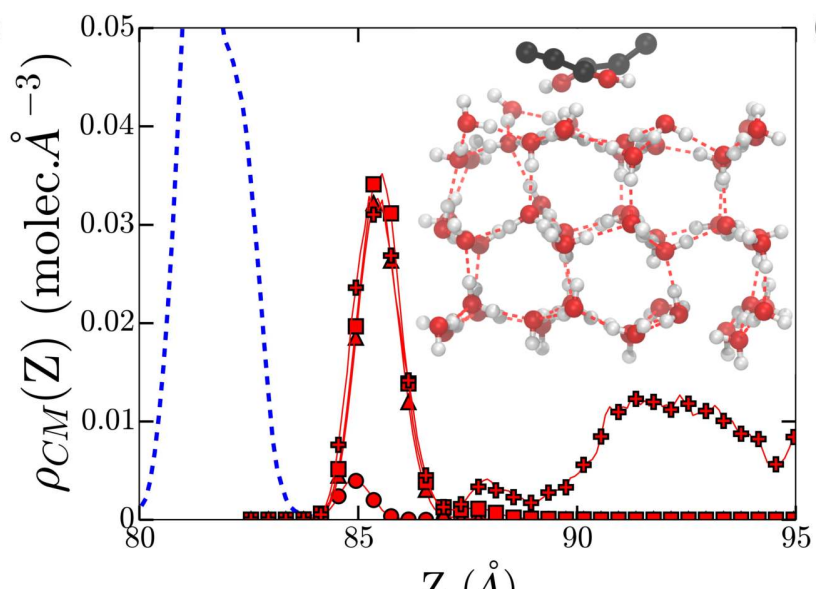

(c)

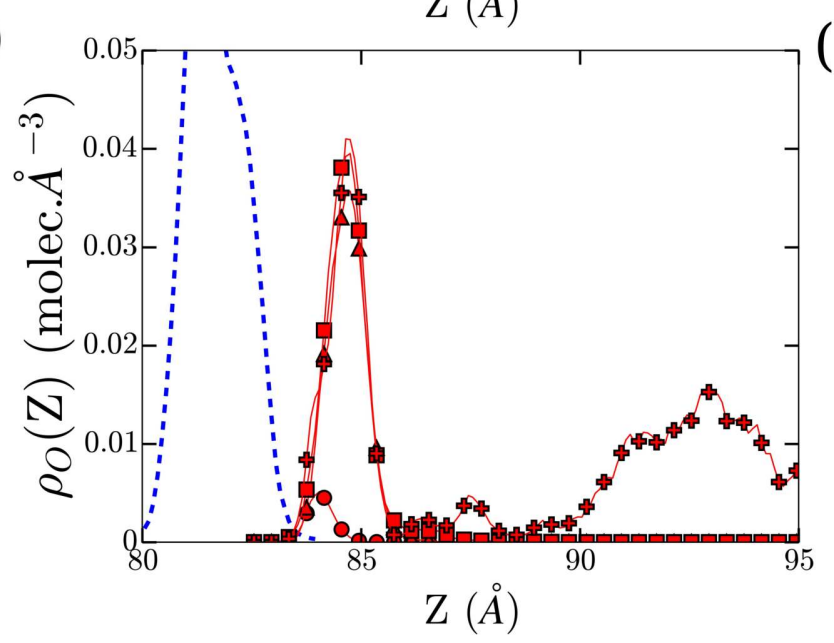

(b)

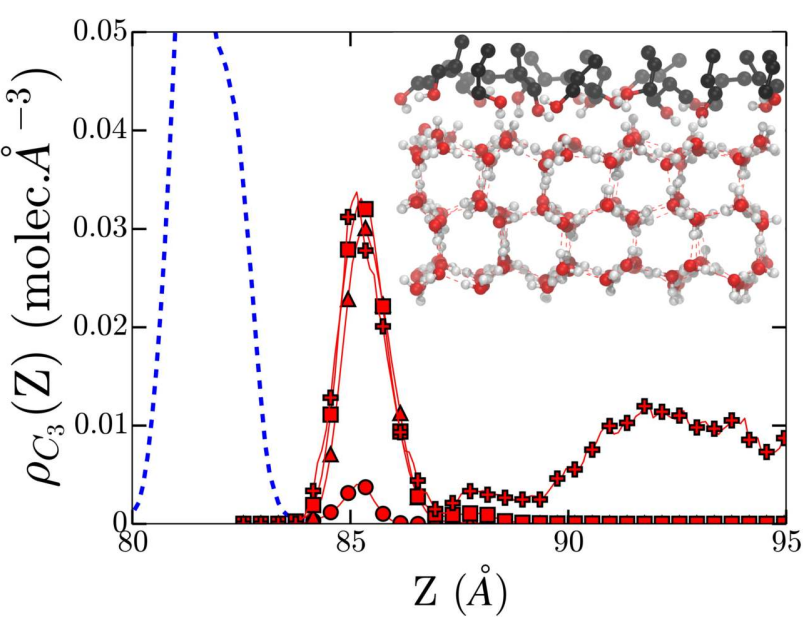

(d)

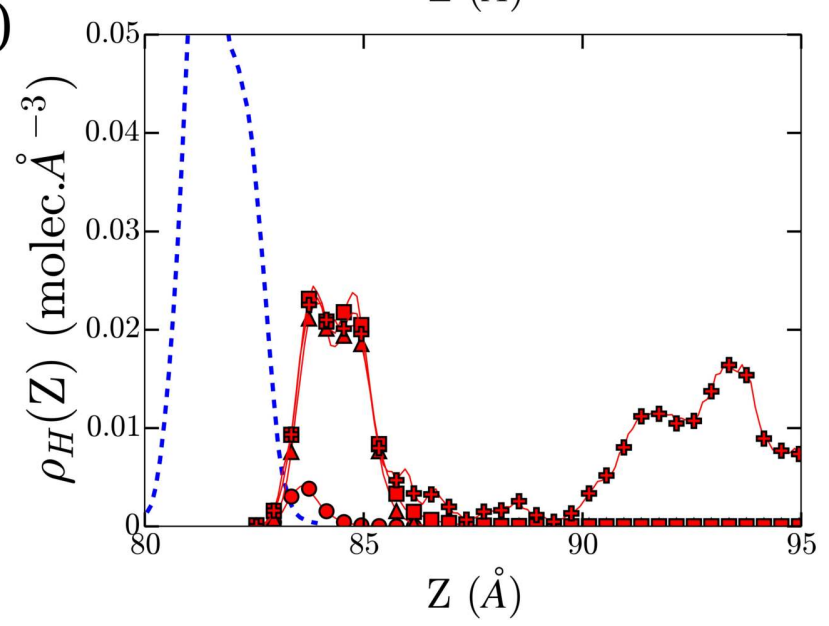

Figure 5: Number density profile of the propanol (a) centres of mass, (b) $\mathrm{C}_{3}$ (c) O and (d) H atoms, in Systems 1 (circles), 2 (triangles), 3 (squares) and 4 (crosses), as obtained from the simulations. For reference, the outer tail of the number density profile of the water centres of mass in System 1 is also given (dashed blue line). All profiles shown are symmetrised over the two surfaces present in the basic simulation box. In addition, snapshots issued from the simulations of Systems 1 (low coverage) and 3 (high coverage) are given in panel (a) and (b), respectively, to illustrate the adsorption geometry of the propanol molecules at various coverages of the ice surface. 
(a)

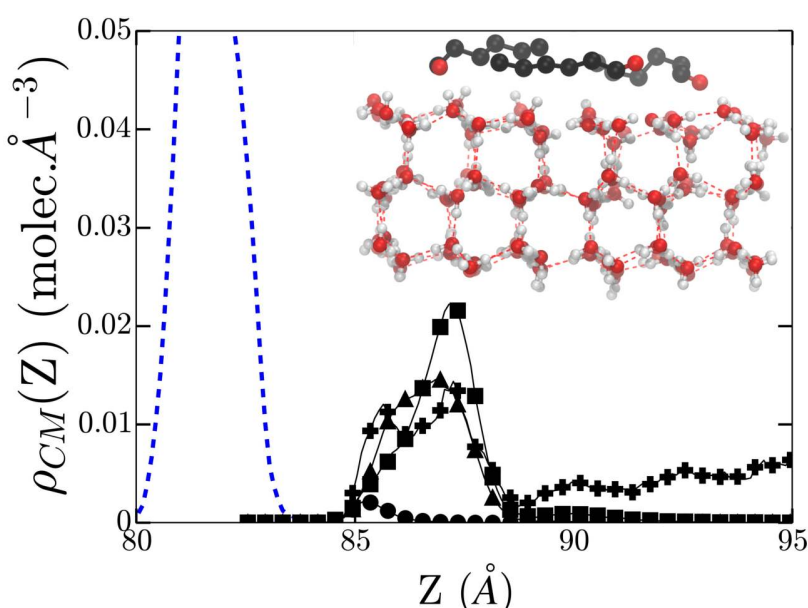

(c)

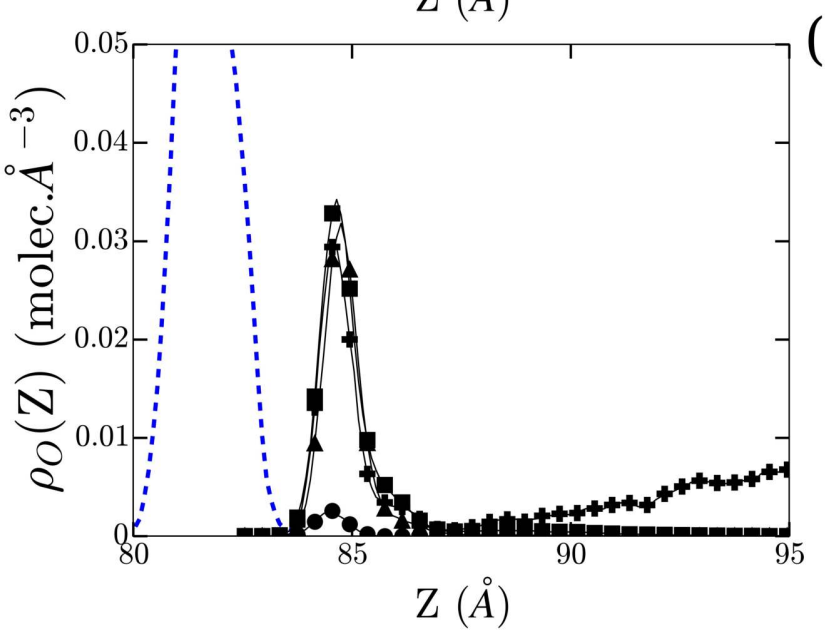

(b)

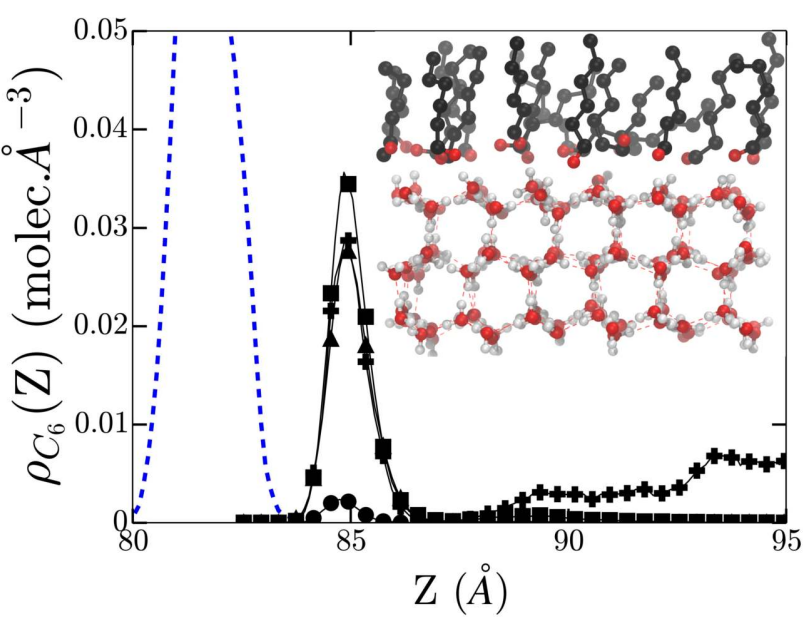

(d)

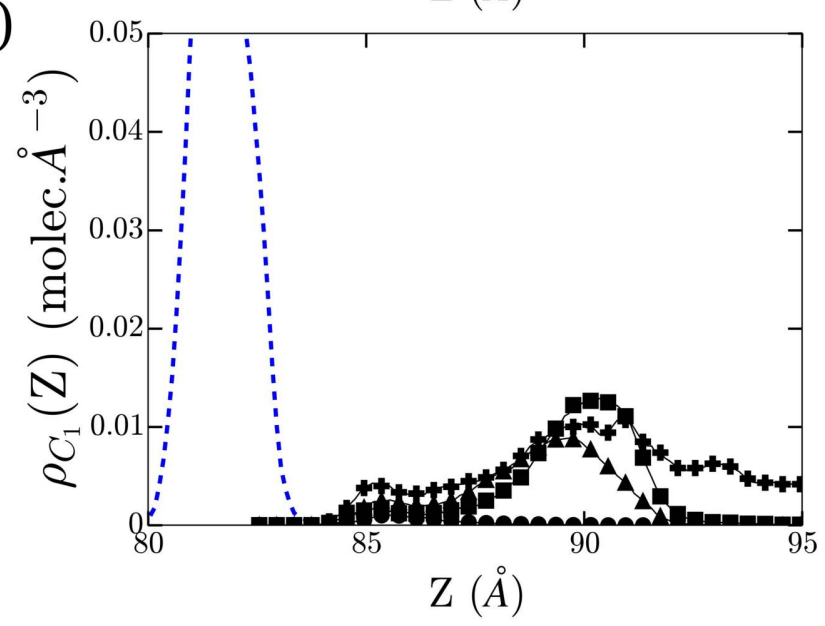

Figure 6: Number density profile of the hexanal (a) centres of mass, (b) $\mathrm{C}_{6}$ (c) $\mathrm{O}$ and (d) $\mathrm{C}_{1}$ atoms, in Systems 1 (circles), 2 (triangles), 3 (squares) and 4 (crosses), as obtained from the simulations. For reference, the outer tail of the number density profile of the water centres of mass in System 1 is also given (dashed blue line). All profiles shown are symmetrised over the two surfaces present in the basic simulation box. In addition, snapshots issued from the simulations of Systems 1 (low coverage) and 3 (high coverage) are given in panel (a) and (b), respectively, to illustrate the adsorption geometry of the hexanal molecules at various coverages of the ice surface. 


\section{Propanol}
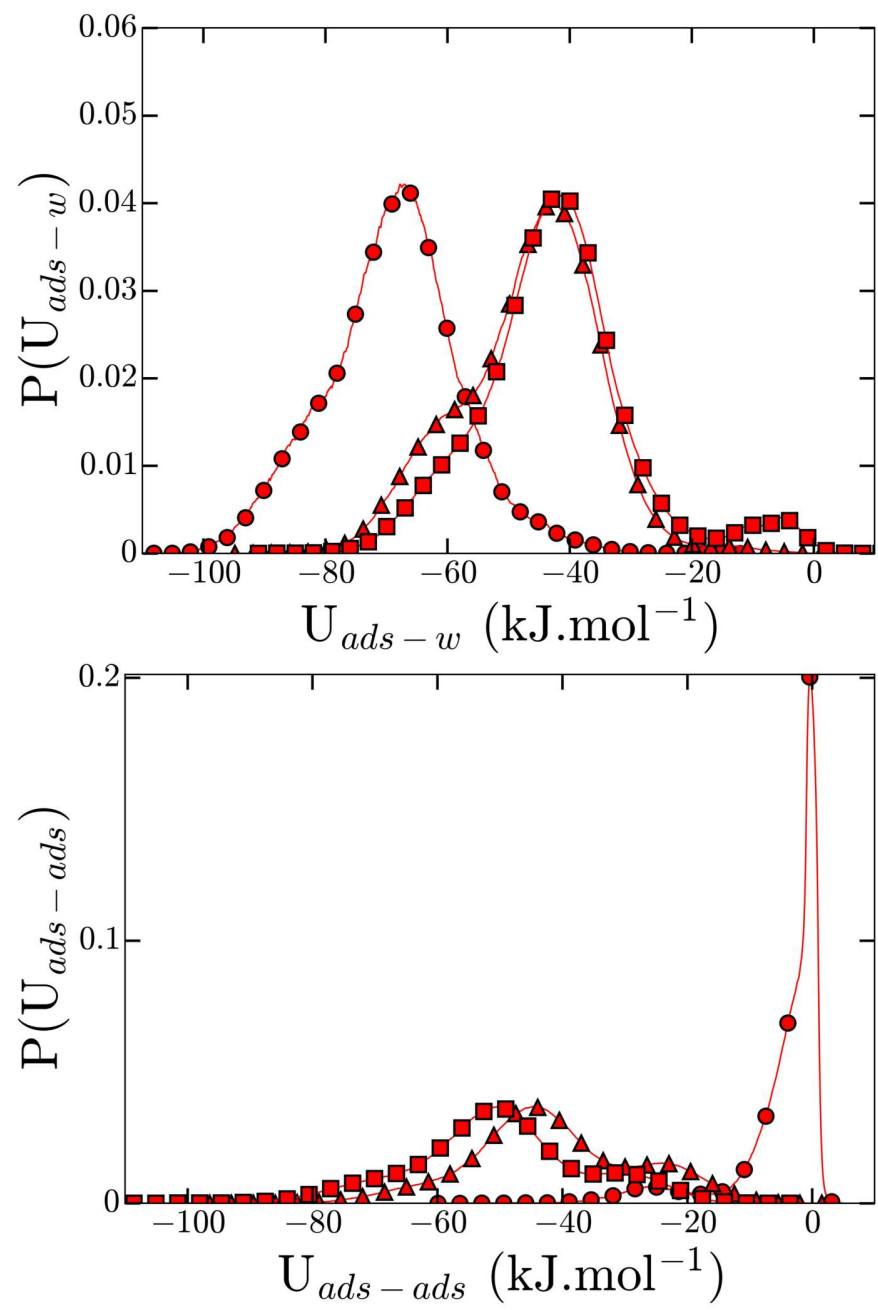

Hexanal
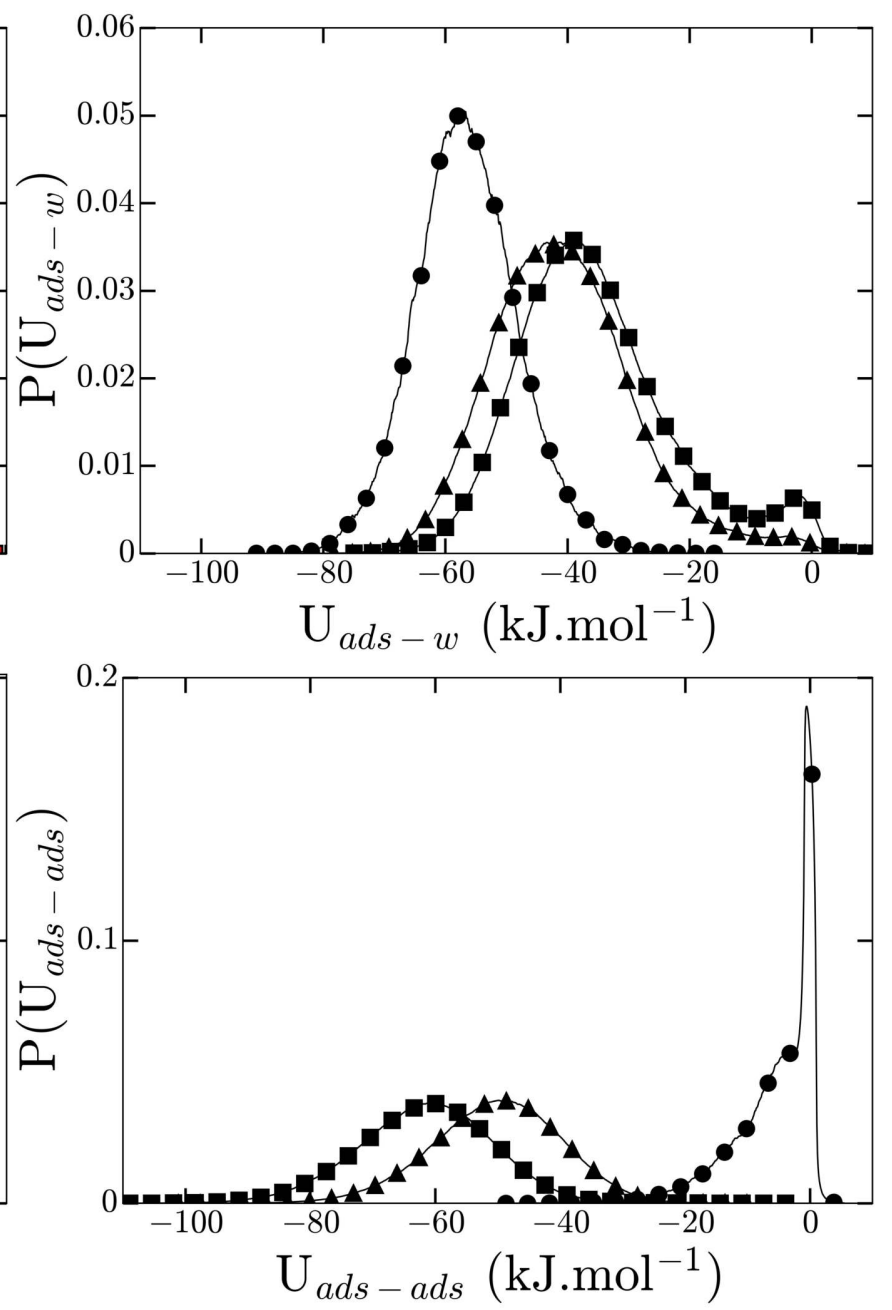

Figure 7: Distribution of the interaction energy of an adsorbed molecule with the ice phase $\left(\mathrm{P}\left(\mathrm{U}_{\text {ads-w }}\right)\right.$, top row $)$ and with the other adsorbed molecules $\left(\mathrm{P}\left(\mathrm{U}_{\text {ads-ads }}\right)\right.$, bottom row) when considering propanol (left hand side, red curves) and hexanal (right hand side, black curves) as adsorbates in the GCMC simulations, for Systems 1 (circles), 2 (triangles) and 3 (squares). 
The $\mathrm{P}\left(\mathrm{U}_{\text {ads-ads }}\right)$ distribution (Figure 7 , bottom) exhibits a large peak at very small energy values in System 1 for both propanol and hexanal adsorbates, reflecting the fact that, at this low coverage, the adsorbed molecules stay rather far from each other at the ice surface. Notice however that the shoulder of this peak towards lower energy is wider for hexanal than for propanol molecules, indicating larger lateral interactions (albeit still weak) within the hexanal adsorbate, even at relatively low coverage. This could explain the different shape of the corresponding isotherms in the low fugacity range (Figure 4). By contrast, at higher coverages of the surface (Systems 2 and 3), the $\mathrm{P}\left(\mathrm{U}_{\text {ads-ads }}\right)$ distribution shifts toward much lower energy values, indicating the strong increase of the lateral interactions within the adsorbate. Just before condensation (i.e. for System 3), this distribution is characterised by a large single peak around $-55 \mathrm{~kJ} / \mathrm{mol}$ in the case of hexanal, whereas it exhibits one main, large peak at around $-50 \mathrm{~kJ} / \mathrm{mol}$ and another, smaller one, around $-25 \mathrm{~kJ} / \mathrm{mol}$, in the case of propanol. Considering the fact that the energy of a hydrogen bond fall roughly in the $[-25--20] \mathrm{kJ} / \mathrm{mol}$ range, the above findings indicates that the adsorbed molecules might form either one or two hydrogen bonds, or only two hydrogen bonds with their neighbours, at monolayer coverage, in the case of propanol and hexanal, respectively. However, the real situation could be more complicated because part of the lateral interactions may come not only from hydrogen bonding between adjacent polar heads, but also from additional interactions between the long aliphatic chains, depending on their respective orientations.

Then, we have calculated the average number of hydrogen bonds formed between propanol and water molecules, and between neighbouring propanol molecules within the adsorbed layer, by using the standard geometrical criterion for the definition of these H-bonds [40]. For System 1, about 3 H-bonds are thus formed between the propanol and the water molecules, in accordance with the calculated value of the heat of adsorption at infinitely low coverage $(-70 \mathrm{~kJ} / \mathrm{mol})$. Upon increasing the propanol coverage with fugacity, the average number of $\mathrm{H}$-bond between propanol and ice decreases from about 3.11 (System 1) to about 1.6 and 1.3 for Systems 2 and 3, respectively, in connection with the increase to about $-40 \mathrm{~kJ} / \mathrm{mol}$ of the corresponding $\mathrm{U}_{\text {ads-w }}$ values. Concomitantly, the average number of $\mathrm{H}$-bonds between neighbouring propanol molecules within the adlayer increases from about 0.1 (System 1) to 0.90 (System 2) and 1.05 (System 3), again in accordance with the variations of the $\mathrm{U}_{\text {ads-ads }}$ values. However, because one $\mathrm{H}$-bond accounts for about $-25 \mathrm{~kJ} / \mathrm{mol}$, only, the peak at around $-50 \mathrm{~kJ} / \mathrm{mol}$ in the $\mathrm{P}\left(\mathrm{U}_{\text {ads-ads }}\right)$ distribution indicates that the aliphatic chains may also significantly contribute to the intra-adsorbate interaction energy. It is worth mentioning that, for hexanal, the interaction model we use do not consider explicitly the $\mathrm{H}$ atom of the aldehyde group, preventing thus any rigorous analysis of the hydrogen bonding for this molecule.

\subsubsection{Orientation of the adsorbed molecules}

The description of the adsorption process can be completed by the characterisation of the molecular orientations at the surface of ice. Here, we focus on the orientation of the molecular axis, which can be defined by the angle $\theta_{1}$ formed between the vector normal to the ice surface $(\mathrm{z})$ and the $\mathrm{C}-\mathrm{C}$ vector joining the $\mathrm{C}$ atom $\left(\mathrm{C}_{1}\right.$, see Figure 1$)$ of the terminal aliphatic group $\left(\mathrm{CH}_{3}\right)$ to the $\mathrm{C}$ atom bearing the alcohol $\left(\mathrm{C}_{3}\right)$ or the aldehyde $\left(\mathrm{C}_{6}\right)$ function. In this case, a value $\theta_{1}=0$ corresponds to a situation where this $\mathrm{C}-\mathrm{C}$ vector is oriented perpendicular to the ice surface, the aliphatic group pointing to the ice surface, while the functional group points to the gas phase. In addition, to get more information on the orientation of the functional groups of these molecules with respect to the ice surface, we also characterise the angle $\theta_{2}$ formed between $\mathrm{z}$ and the $\mathrm{CO}$ vector and, for propanol, the angle $\theta_{3}$ formed between $\mathrm{z}$ and the $\mathrm{OH}$ vector. Note that because the adsorbed molecules are flexible in the simulations, their orientation cannot be unambiguously described by the two independent polar coordinates $\theta$ and $\phi$ that are usually used when considering the orientation of a rigid body relative to an external direction [41].

The following orientational analysis is limited to the propanol and hexanal molecules that are directly attached to the ice surface, i.e. those corresponding to the first peak in the centre of mass density profiles of the adsorbate shown in Figures 5 and 6 . The orientation distribution of the angle $\theta_{1}$ for propanol given in Figure 8 (top left panel) clearly indicates that a reorientation of the molecular axis occurs upon the adsorption of an increasing number of molecules. Indeed, at very low coverage, this distribution is characterised by a single peak with a maximum at the value $\theta_{1} \approx 103^{\circ}$ which shifts to larger values upon coverage increase, up to a maxi- mum corresponding to $\theta_{1} \approx 155^{\circ}$. The propanol molecules thus move from a configuration nearly parallel to the ice surface at low coverage, to a configuration in which their molecular axis is tilted from the surface normal by about $25^{\circ}$, the polar head pointing to the ice surface, at higher coverages. Meanwhile, the orientation of the polar head significantly changes upon adsorption of an increasing number of propanol molecules, as indicated by the evolution of the distributions of $\theta_{2}$ and $\theta_{3}$ angles. Interestingly, at high coverage, $\mathrm{P}\left(\theta_{3}\right)$ is characterised by a double peak (bottom left panel of Figure 8) which corresponds to configurations where the $\mathrm{OH}$ 
bond points either down to the surface or to the gas phase, supporting the assumption on the formation of proton donor and proton acceptor hydrogens bonds with the underlying water molecules, as suggested by the analysis of the density profile for $\mathrm{H}$ atoms (see above).

A similar behaviour is observed for the hexanal molecules that move from configurations nearly parallel to the ice surface at low coverage (right top panel of Figure 8) to configurations in which their molecular axis is tilted from the surface normal by about $30^{\circ}$, so that the aldehyde function points to the ice surface at higher coverages, the long aliphatic chain being oriented in such a way that the terminal $\mathrm{CH}_{3}$ points towards the gas phase (see also the snapshots given in Figure 6 for illustration). Note however that the orientation of the polar head remains unchanged irrespective of the coverage, the $\mathrm{CO}$ bond being always preferentially nearly parallel to the ice surface, as indicated by the corresponding distribution $\mathrm{P}\left(\theta_{2}\right)$ (bottom right panel of Figure 8).

The results of this orientational analysis are thus in clear accordance with the information issued from the evolutions of the density profiles upon coverage increase and of the corresponding distributions of the $\mathrm{U}_{\text {ads-ads }}$ and $\mathrm{U}_{\text {ads-w }}$ contributions to the total binding energy, as analysed above.

\section{Discussion}

The results of the present GCMC simulations have evidenced that ethylene is quite weakly bonded to ice, with a mean interaction energy of about $-9 \mathrm{~kJ} / \mathrm{mol}$, i.e. $-0.093 \mathrm{eV}$, with the ice surface, which precludes the formation of any stable adlayer on ice before 3D condensation occurs. Although there is no experimental information at $236 \mathrm{~K}$, the results of the simulation agree quite well with previous experimental conclusions [42]. Indeed, on the basis of infrared spectroscopy measurements, it has been concluded that the interaction energy of ethylene on amorphous ice is equal to about $0.16 \mathrm{eV}(15 \mathrm{~kJ} / \mathrm{mol})$, which corresponds to a measured shift of $75 \mathrm{~cm}^{-1}$ of the dangling-H signal. Because this shift has been measured to be $1 / 3$ less on crystalline ice $\left(50 \mathrm{~cm}^{-1}\right)$, and assuming a linear relation between shift and bond energy [42], we can infer that the corresponding interaction between ethylene and crystalline ice would be proportionally smaller than on amorphous ice, with a value thus estimated to be about $0.11 \mathrm{eV}$, i.e. $10.6 \mathrm{~kJ} / \mathrm{mol}$, in fair agreement with the value issued from our simulations. Moreover, the experimental spectra do not give any evidence of ethylene acting as proton donor on ice [42], which is consistent with the flat orientation of the molecular axis found in the present GCMC simulations.
By contrast, GCMC simulations have revealed that both propanol and hexanal molecules can be strongly adsorbed at the surface of crystalline ice at $236 \mathrm{~K}$, a temperature typical of Enceladus' environment. The corresponding adsorption process is characterised by the stabilisation of a single layer of propanol molecules over a large range of fugacity, and by the progressive saturation of all possible adsorption sites for hexanal, up to the 3D condensation of the adsorbate above the ice phase. These features are in qualitative agreement with experimental conclusions coming from flow tube measurements by Sokolov and Abbatt at $228 \mathrm{~K}$ [26]. Indeed, in these experiments, it has been concluded that the adsorption process of 1-propanol on ice follows Langmuir behaviour and that, in the pressure range investigated, propanol may form a saturated layer at the surface of ice. Moreover, there are strong indications that the adsorbed molecules are oriented in such a way that their hydroxyl group binds to the ice surface [26], in accordance with the results of the present orientational analysis. Notice however, that the simulated isotherm clearly deviates from the Langmuir behaviour, even at relatively moderate pressure, and this deviation could be related to the increasing contribution of the lateral interactions within the adsorbed layer [37]. This indicates that, for molecules that may strongly interact together even at moderate coverage of the ice surface, experiments should be also conducted (if possible) under relatively high partial pressure conditions. Nevertheless, the adsorption enthalpy of propanol on ice at low coverage has been estimated to be around $-68 \mathrm{~kJ} / \mathrm{mol}(-0.7 \mathrm{eV})$ in the experiments [26, 27], a value which is very close to the one determined in the present GCMC simulations for System 1 (see above).

For hexanal, the uptake at the surface of ice has been shown to exhibit a linear behaviour between 218 and $226 \mathrm{~K}$, in the pressure range experimentally investigated $(\mathrm{P}<0.013 \mathrm{~Pa})$, so that no Langmuir equation can be adequately fit on the data of the corresponding measured isotherms [26], in qualitative agreement with the non-Langmuir behaviour evidenced by the results of the present GCMC calculations at $236 \mathrm{~K}$. Meanwhile, the adsorption energy of hexanal has been experimentally determined to be around $-64.8 \pm 5.8 \mathrm{~kJ} / \mathrm{mol}$ $(-0.67 \pm 0.06 \mathrm{eV})[26]$, i.e. only slightly lower than the value calculated here using Monte Carlo simulations $(-0.61 \mathrm{eV})$.

All these features give thus confidence in the ability of the interaction potential models used in the present GCMC simulations, to accurately represent the adsorption process of ethylene, propanol and hexanal molecules at the surface of hexagonal ice, in thermodynamics conditions relevant for Enceladus' environments. As a consequence, the results of such studies can be used to give reliable input data for 
Propanol
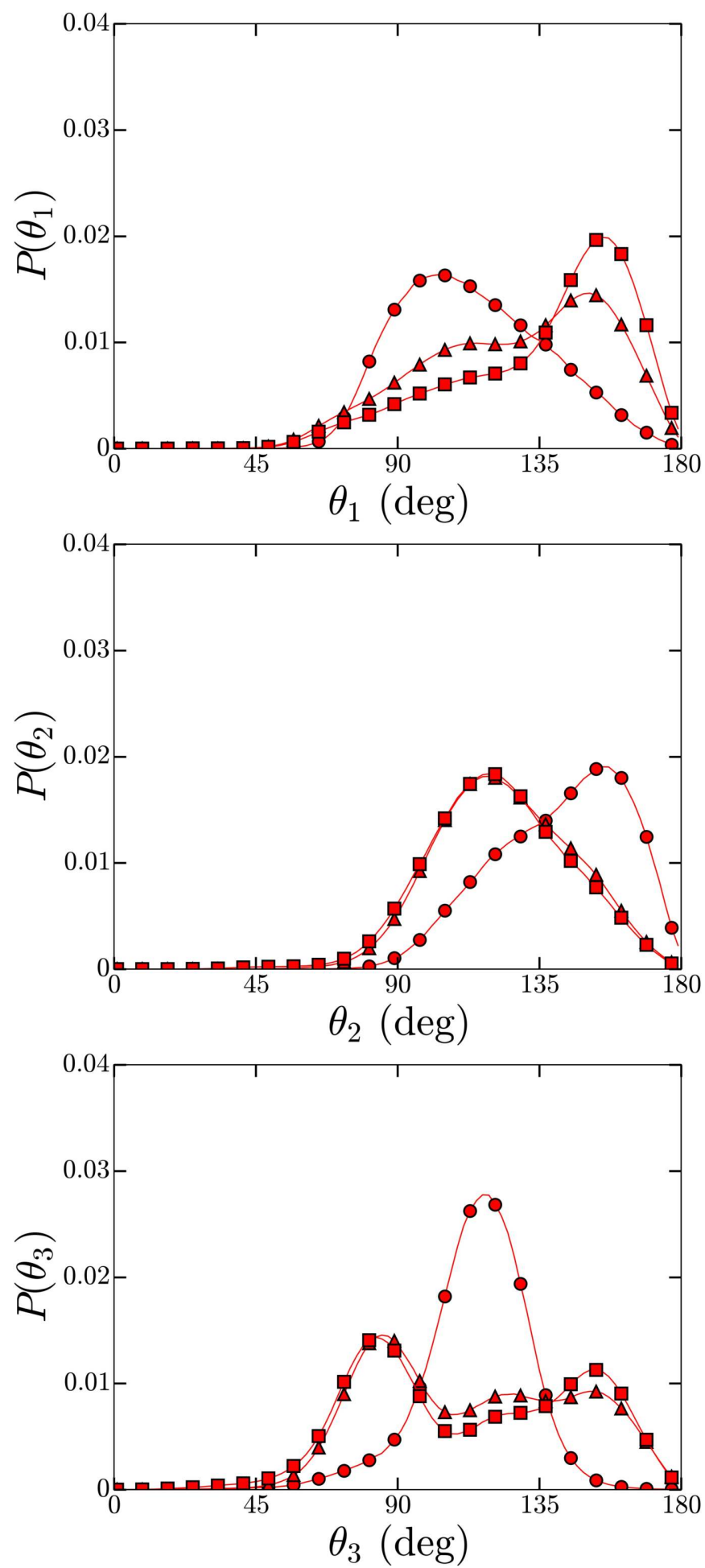

Hexanal
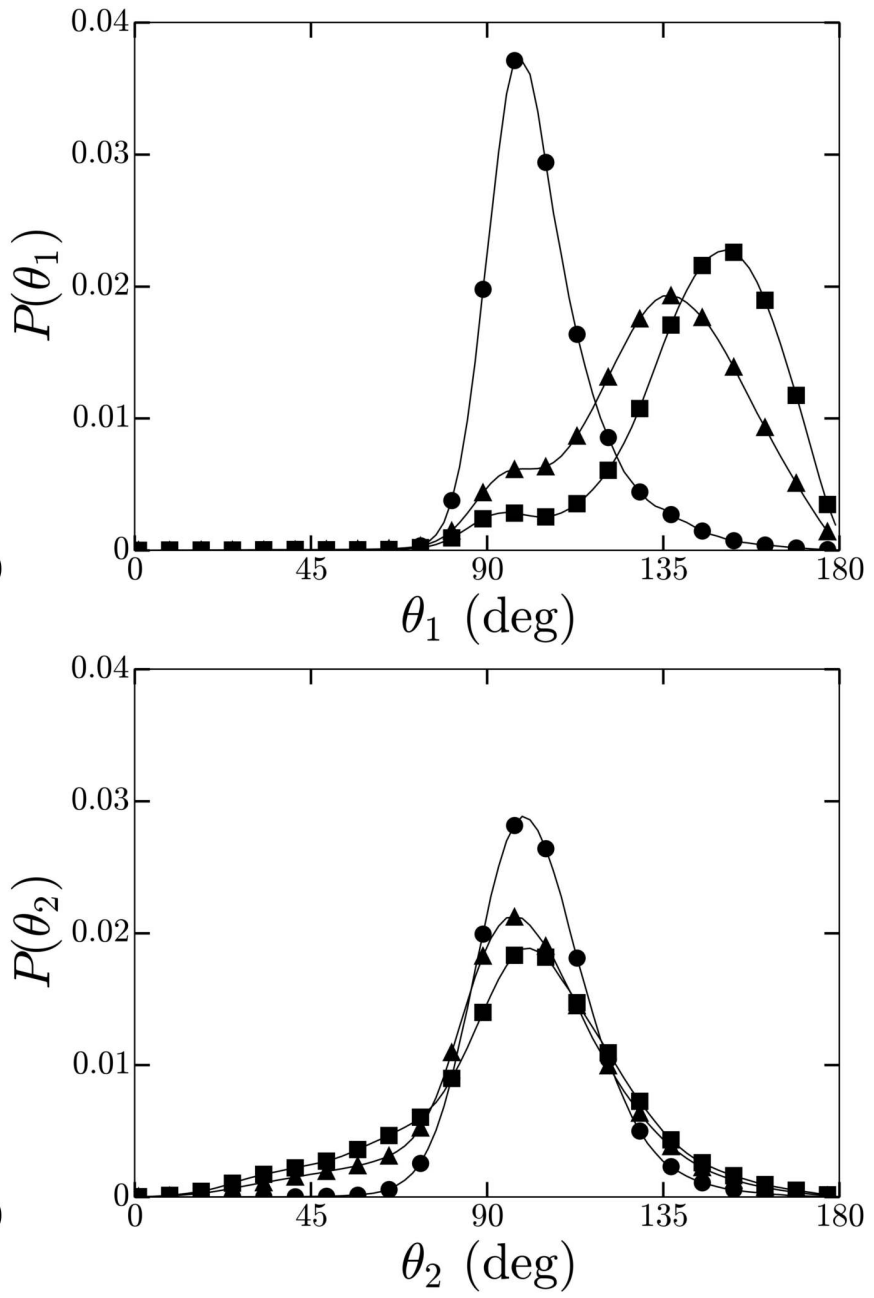

Figure 8: Distribution of the molecular orientations for propanol (red curves) and hexanal (black curves) molecules adsorbed on ice, for Systems 1 (circles), 2 (triangles), and 3 (squares). Top row: $\theta_{1}$ is the angle formed between the surface normal to the ice surface $(\mathrm{z})$ and the $\mathrm{C}-\mathrm{C}$ vector joining the $\mathrm{C}$ atom $\left(\mathrm{C}_{1}\right.$, see Figure 1$)$ of the terminal aliphatic group $\left(\mathrm{CH}_{3}\right)$ to the $\mathrm{C}$ atom bearing the alcohol $\left(\mathrm{C}_{3}\right.$, see Figure 1$)$ or the aldehyde function $\left(\mathrm{C}_{6}\right.$, see Figure 1). Middle row: $\theta_{2}$ is the angle formed between $\mathrm{z}$ and the $\mathrm{CO}$ vector; bottom row (for propanol, only), $\theta_{3}$ is the angle formed between $\mathrm{z}$ and the $\mathrm{OH}$ vector. 
adsorption models such as the one recently developed by Bouquet et al. [5]. This seems particularly true when considering the binding energy at low coverage, which has been shown to be the key parameter in this model, for segregating between compounds that are trapped or released by the ice surface. Thus, according to the conclusions of Bouquet et al., ethylene which has a binding energy greater than $-0.5 \mathrm{eV}$ should be negligibly present at the ice surface, in contrast with propanol which has a binding energy of about $-0.7 \mathrm{eV}$. The adsorption energy of hexanal falling just in between these two values, this species may compete with compounds of lower energy and may thus likely stay at the ice surface in some situations [5].

Moreover, the present simulations indicate that the shape of the adsorption isotherm of propanol and hexanal significantly deviates from the Langmuir equation unless only the very low pressure range is taken into account when fitting the corresponding parameters (for propanol). This non-Langmuir behaviour influences the value of the saturation coverage, which thus could be underestimated in the interpretation of the experimental data, with respect to the value calculated in the GCMC simulations when the lateral interactions are taken into account. For instance, this saturation coverage has been experimentally estimated to be around $3.1 \times 10^{14}$ molecules.cm ${ }^{-2}$ for propanol at 226 $\mathrm{K}$, whereas the present simulation results indicate that it is certainly greater than $4.3 \times 10^{14}$ molecules.cm ${ }^{-2}$ at $236 \mathrm{~K}$, i.e. the value issued from our Langmuir fit (see Figure 4, and the deviation from the Langmuir behaviour at high relative pressure). It is thus reasonable to conclude, as previously [37], that this saturation coverage is likely a meaningless parameter in experimental studies of trace gas-ice interactions conducted only at low pressures, when considering adsorbates for which lateral interactions are non-negligible. This emphasises the need for numerical data issued from, for instance, GCMC simulations based on accurate interaction potential models, to achieve a correct determination of the saturation coverage of organic compounds on ice, when the corresponding experimental data are poorly defined.

Worthwhile to note is that, in addition to the binding energy and saturation coverage, GCMC simulations give also useful information on the orientations of the adsorbed molecules, which is an important parameter for studying chemical reactions that can occur at the surface of ice. This appears crucial especially when addressing the ability of ice surfaces at promoting prebiotic chemistry in Space [42], or at influencing on oxidation processes of organic compounds in the troposphere [43].

\section{Conclusions}

In this paper, the adsorption of ethylene, propanol and hexanal molecules on crystalline ice has been characterised, for the first time, by computer simulations performed at $236 \mathrm{~K}$, a temperature which is typical of Enceladus' environments. The results of the simulations turned out to be in good agreement with the major features provided by the available experiments on similar systems, especially at low coverage when the lateral interactions within the adsorbed layer do not play any significant role.

In analysing the properties of the adsorption layer formed by propanol and hexanal adsorbates at the surface of ice, we have found that their molecularlevel structures change upon saturation. Indeed, at low surface coverage, the adsorption is driven by the interaction of the organic molecules with the ice phase and, as a consequence, the adsorbed propanol and hexanal molecules lie more or less parallel to the ice surface. On the other hand, upon saturation, the adsorbate-adsorbate interactions become more and more important and the molecular axis tend to become tilted with respect to the surface normal by about $25-30^{\circ}$. Nevertheless, the polar head of these molecules always stay attached to the ice surface, irrespective of the coverage.

More interestingly, the results of the present simulations confirm that the binding energy to ice of propanol and hexanal has values falling either in (for hexanal) or at the limit of (in the case of propanol) the intermediate energy range (between 0.5 and 0.7 $\mathrm{eV}$ ) for which the model of Bouquet et al. [5] indicates that the organic compounds may be present on the ice surface only if the competition to occupy adsorption sites allows. In addition, considering that the saturation vapour pressure of water ice at the wall's temperature of the ice cracks has been estimated to be about $17 \mathrm{~Pa}$ at $236 \mathrm{~K} \mathrm{[5]} \mathrm{and} \mathrm{that} \mathrm{the} \mathrm{organic} \mathrm{com-}$ pounds accounts for a few tenth of percent of the gas phase composition [6], we can infer that only the first part of the propanol and hexanal adsorption isotherms, calculated at low fugacity, likely fits the real conditions of the ice cracks on Enceladus. Consequently, if more quantitative data on the possible competitive adsorption process on ice are required, further studies should focus especially on the very low coverage stage of the adsorption process. GCMC simulations taking into account a gas phase containing various mixtures of both propanol and hexanal molecules could be thus envisaged, following the approach recently developed to characterise the selective trapping of $\mathrm{CO}$ and $\mathrm{N}_{2}$ by clathrate hydrates and various ice surfaces $[21,22$, 23]. Work in that direction is currently in progress. 


\section{Acknowledgements}

The authors gratefully acknowledge financial support from the Region Bourgogne Franche-Comté and from the Observatoire des Sciences de l'Univers OSU THETA (France). Computations have been performed on the supercomputer facilities of the Mésocentre de calcul de Franche-Comté.

\section{References}

[1] C.C. Porco et al. "Cassini Observes the Active South Pole of Enceladus". In: Science (New York, N.Y.) 311 (Apr. 2006), pp. 1393-401. DOI: 10. 1126/science.1123013.

[2] J Hunter Waite et al. "Cassini ion and neutral mass spectrometer: Enceladus plume composition and structure". In: science 311.5766 (2006), pp. 1419-1422. DOI: 10.1126/science. 1121290.

[3] Candice J Hansen et al. "Enceladus' water vapor plume". In: Science 311.5766 (2006), pp. 14221425. DOI: $10.1126 /$ science. 1121254.

[4] Sascha Kempf, Uwe Beckmann, and Jürgen Schmidt. "How the Enceladus dust plume feeds Saturn's E ring". In: Icarus 206.2 (2010), pp. 446-457. DOI: https://doi.org/10.1016/ j.icarus.2009.09.016.

[5] Alexis Bouquet, Christopher Glein, and J. Waite. "How Adsorption Affects the Gas-Ice Partitioning of Organics Erupted from Enceladus". In: The Astrophysical Journal 873 (Feb. 2019), p. 28. DOI: $10.3847 / 1538-4357 / \mathrm{ab} 0100$.

[6] N Khawaja et al. "Low-mass nitrogen-, oxygenbearing, and aromatic compounds in Enceladean ice grains". In: Monthly Notices of the Royal Astronomical Society 489.4 (2019), pp. 52315243. DOI: https://doi.org/10.1093/mnras/ stz2280.

[7] T. Bartels-Rausch et al. "A review of air-ice chemical and physical interactions (AICI): liquids, quasi-liquids, and solids in snow". In: $A t$ mospheric Chemistry and Physics 14.3 (2014), pp. 1587-1633. DOI: $10.5194 /$ acp $-14-1587$ 2014.

[8] Jonathan PD Abbatt. "Interactions of atmospheric trace gases with ice surfaces: Adsorption and reaction". In: Chemical reviews 103.12 (2003), pp. 4783-4800. DOI: https://doi.org/ $10.1021 / \operatorname{cr} 0206418$.
[9] JPD Abbatt et al. "Halogen activation via interactions with environmental ice and snow in the polar lower troposphere and other regions". In: Atmospheric Chemistry and Physics 12.14 (2012), pp. 6237-6271. DOI: https://doi.org/ 10.5194/acp-12-6237-2012.

[10] Thomas Huthwelker, Markus Ammann, and Thomas Peter. "The Uptake of Acidic Gases on Ice". In: Chemical reviews 106 (May 2006), pp. 1375-444. DOI: $10.1021 /$ cr020506v.

[11] Florent Dominé and Paul B Shepson. "Air-snow interactions and atmospheric chemistry". In: Science 297.5586 (2002), pp. 1506-1510. DOI: 10. $1126 /$ science. 1074610.

[12] John N Crowley et al. "Evaluated kinetic and photochemical data for atmospheric chemistry: Volume V-heterogeneous reactions on solid substrates". In: Atmospheric Chemistry and Physics 10.18 (2010), pp. 9059-9223. DOI: https://doi. org/10.5194/acp-10-9059-2010.

[13] V Faye McNeill et al. "Organics in environmental ices: sources, chemistry, and impacts". In: Atmospheric Chemistry and Physics 12.20 (2012), pp. 9653-9678. DOI: https : // doi .org/10. 5194/acp-12-9653-2012.

[14] WA Schutte. "Laboratory simulation of physical and chemical processes in interstellar ices". In: Symposium-International Astronomical Union. Vol. 178. Cambridge University Press. 1997, pp. 331-340. DOI: https://doi.org/10.1017/ S0074180900009475.

[15] BA Magee and JH Waite. "Neutral gas composition of Enceladus' plume-model parameter insights from Cassini-INMS". In: Lunar and planetary science conference. 1964. 2017, p. 2974.

[16] S Picaud et al. "Molecular dynamics and Monte Carlo simulations of organic compounds adsorbed on ice surfaces". In: AIP Conference Proceedings. Vol. 963. 1. American Institute of Physics. 2007, pp. 296-307. DOI: https://doi. org/10.1063/1.2827013.

[17] G Hantal P Jedlovszky LB Partay and S Picaud PNM Hoang. "Molecular-scale modeling of the adsorption of partially oxidized organic compounds on ice." In: Trends in Physical Chemistry 13 (2008), pp. 1-19.

[18] Sylvain Picaud and Pál Jedlovszky. "Molecularscale simulations of organic compounds on ice: application to atmospheric and interstellar sciences". In: Molecular Simulation 45.4-5 (2019), pp. 403-416. DOI: https://doi.org/10.1080/ 08927022.2018 .1502428$. 
[19] S Lectez et al. "A 32-70 K Formation Temperature Range for the Ice Grains Agglomerated by Comet $67 \mathrm{P} /$ Churyumov-Gerasimenko". In: The Astrophysical Journal Letters 805.1 (2015), p. L1. DOI: $10.1088 / 2041-8205 / 805 / 1 / L 1$.

[20] Balázs Fábián et al. "Ammonia clathrate hydrate as seen from grand canonical monte carlo simulations". In: ACS Earth and Space Chemistry 2.5 (2018), pp. 521-531. DOI: https://doi. org/10.1021/acsearthspacechem. 7b00133.

[21] Antoine Patt et al. "A grand canonical monte carlo study of the N2, CO, and mixed N2-CO clathrate hydrates". In: The Journal of Physical Chemistry C 122.32 (2018), pp. 18432-18444. DOI: https : //doi .org/10.1021/acs . jpcc . $8 \mathrm{~b} 03657$.

[22] C Petuya et al. "Molecular Selectivity of CON2 Mixed Hydrates: Raman Spectroscopy and GCMC Studies". In: The Journal of Physical Chemistry C 124.22 (2020), pp. 11886-11891. DOI: https : //doi .org/10.1021/acs . jpcc . 0c01315.

[23] Antoine Patt et al. "Adsorption of CO and N2 molecules at the surface of solid water. A grand canonical Monte Carlo study". In: The Journal of Chemical Physics 153.20 (2020), p. 204502. DOI: https://doi.org/10.1063/5.0031254.

[24] SC Silva and J Paul Devlin. "Interaction of acetylene, ethylene, and benzene with ice surfaces". In: The Journal of Physical Chemistry 98.42 (1994), pp. 10847-10852. DOI: 10.1021/j100093a027.

[25] DJ Donaldson and Darren Anderson. "Adsorption of atmospheric gases at the air- water interface. 2. C1- C4 alcohols, acids, and acetone". In: The Journal of Physical Chemistry A 103.7 (1999), pp. 871-876. DOI: https://doi.org/10. 1021/jp983963h.

[26] O. Sokolov and J. P. D. Abbatt. "Adsorption to Ice of n-Alcohols (Ethanol to 1-Hexanol), Acetic Acid, and Hexanal". In: The Journal of Physical Chemistry A 106.5 (2002), pp. 775-782. DOI: 10.1021/jp013291m.

[27] V Buch, P Sandler, and J Sadlej. Simulations of H2O solid, liquid, and clusters, with an emphasis on ferroelectric ordering transition in hexagonal ice. 1998. DOI: https ://doi .org/10.1021/ jp980866f.

[28] John D Bernal and Ralph H Fowler. "A theory of water and ionic solution, with particular reference to hydrogen and hydroxyl ions". In: The Journal of Chemical Physics 1.8 (1933), pp. 515-548. DOI: https://doi.org/10.1063/ 1.1749327.
[29] J.L.F. Abascal et al. "A potential model for the study of ices and amorphous water: TIP4P/Ice". In: The Journal of chemical physics 122 (July 2005), p. 234511. DOI: 10.1063/1.1931662.

[30] Emeric Bourasseau et al. "New optimization method for intermolecular potentials: Optimization of a new anisotropic united atoms potential for olefins: Prediction of equilibrium properties". In: The Journal of Chemical Physics 118 (Feb. 2003), pp. 3020-3034. DOI: 10.1063/1.1537245.

[31] Nicolas Ferrando et al. "Transferable Force Field for Alcohols and Polyalcohols". In: The journal of physical chemistry. B 113 (May 2009), pp. 5985-95. DOI: 10.1021/jp810915z.

[32] Nicolas Ferrando, Véronique Lachet, and Anne Boutin. "Monte Carlo Simulations of Mixtures Involving Ketones and Aldehydes by a Direct Bubble Pressure Calculation". In: The journal of physical chemistry. B 114 (July 2010), pp. 86808. DOI: $10.1021 / j p 1031724$.

[33] M. P. Allen and D. J. Tildesley. Computer Simulation of Liquids. 1st. USA: Clarendon Press, 1987. ISBN: 0198556454.

[34] Philippe Ungerer, Bernard Tavitian, and Anne Boutin. Applications of molecular simulation in the oil and gas industry: Monte Carlo methods. Editions Technip, 2005.

[35] PJ Linstrom and WG Mallard. NIST Chemistry WebBook, NIST Standard Reference Database Number 69. DOI: https://doi.org/10.18434/ T4D303.

[36] György Hantal et al. "Calculation of the adsorption isotherm of formaldehyde on ice by grand canonical Monte Carlo simulation". In: The Journal of Physical Chemistry C 111.38 (2007), pp. 14170-14178. DOI: https : // doi . org/10.1021/jp0742564.

[37] Pál Jedlovszky et al. "Determination of the Adsorption Isotherm of Methanol on the Surface of Ice. An Experimental and Grand Canonical Monte Carlo Simulation Study". In: Journal of the American Chemical Society 128.47 (2006). PMID: 17117883, pp. 15300-15309. DOI: $10.1021 / \mathrm{ja} 065553+$.

[38] Mélanie Petitjean et al. "Adsorption of Benzaldehyde at the Surface of Ice, Studied by Experimental Method and Computer Simulation". In: Langmuir 26.12 (2010). PMID: 20329716, pp. 9596-9606. DOI: 10.1021/la100169h. 
[39] Mária Darvas et al. "Adsorption of Acetaldehyde on Ice As Seen from Computer Simulation and Infrared Spectroscopy Measurements". In: Langmuir 28.9 (2012). PMID: 22320190, pp. 41984207. DOI: $10.1021 / \mathrm{la} 204472 \mathrm{k}$.

[40] Elangannan Arunan et al. "Definition of the hydrogen bond (IUPAC Recommendations 2011)". In: Pure and applied chemistry 83.8 (2011), pp. 1637-1641. DOI: 10 . 1351 / PAC - REC - 1001-02.

[41] Pál Jedlovszky, Árpád Vincze, and George Horvai. "New insight into the orientational order of water molecules at the water/1,2-dichloroethane interface: A Monte Carlo simulation study". In: The Journal of Chemical Physics 117.5 (2002), pp. 2271-2280. DOI: 10.1063/1.1488579.

[42] S. C. Silva and J. Paul Devlin. "Interaction of Acetylene, Ethylene, and Benzene with Ice Surfaces". In: The Journal of Physical Chemistry 98.42 (1994), pp. 10847-10852. DOI: 10.1021/ j100093a027.

[43] Kitti Korcsok et al. "Multiscale Modeling of Interfacial Oxidation Mechanism at Air/Organic Interface: Reactions of $\mathrm{CH} 2=\mathrm{CH}$-Terminated Self-Assembled Monolayer with OH, O3, and HO2". In: The Journal of Physical Chemistry C 122.18 (2018), pp. 9886-9898. DOI: 10.1021/ acs.jpcc. 8b00071. 